\title{
openheart Mechanisms of ECG signs in chronic obstructive pulmonary disease
}

\author{
Marte Strømsnes Larssen, ${ }^{1,2}$ Kjetil Steine, ${ }^{1,3}$ Janne Mykland Hilde, ${ }^{3}$ \\ Ingunn Skjørten, ${ }^{4}$ Christian Hodnesdal, ${ }^{1,2}$ Knut Liestøl, ${ }^{5}$ Knut Gjesdal ${ }^{1,2}$
}

\begin{abstract}
- Additional material is published online only. To view please visit the journal online (http://dx.doi.org/10.1136/ openhrt-2016-000552).
\end{abstract}

To cite: Larssen MS, Steine K, Hilde JM, et al. Mechanisms of ECG signs in chronic obstructive pulmonary disease. Open Heart 2017;0:e000552. doi:10.1136/ openhrt-2016-000552

Received 21 October 2016 Revised 29 December 2016 Accepted 3 January 2017

\section{(a) CrossMark}

${ }^{1}$ Faculty of Medicine, University of Oslo, Oslo, Norway 2Department of Cardiology Ullevål, Oslo University Hospital, Oslo, Norway

${ }^{3}$ Department of Cardiology, Akershus University Hospital, Oslo, Norway

${ }^{4}$ LHL-Clinics, Glittre, Hakadal, Norway

${ }^{5}$ Faculty of Mathematics and Natural Sciences, University of Oslo, Oslo, Norway

\section{Correspondence to}

Professor Knut Gjesdal; knut. gjesdal@medisin.uio.no

\begin{abstract}
Objective Patients with chronic obstructive pulmonary disease (COPD) often have abnormal ECGs. Our aim was to separate the effects on ECG by airway obstruction, emphysema and right ventricular (RV) afterload in patients with COPD.
\end{abstract}

Methods A cross-sectional study was performed on 101 patients with COPD without left heart disease and 32 healthy age-matched controls. Body mass index (BMI) was measured, and pulmonary function tests, ECG, echocardiography and right heart catheterisation (only patients) were performed. Variables were grouped into (1) airway obstruction by FEV\% (percentage of forced expiratory volume)_predicted, (2) emphysema by residual volume/total lung capacity and residual volume (percent of predicted) and (3) RV afterload by mean pulmonary pressure, artery compliance, vascular resistance and RV wall thickness.

Results In multivariate regression analysis, emphysema correlated negatively to $\mathrm{R}+\mathrm{S}$ amplitudes in horizontal and frontal leads, RV/left ventricle (LV) end-diastolic volume ratio to horizontal amplitudes and BMI negatively to frontal amplitudes. Increased airway obstruction, RV afterload and BMI correlated with horizontal QRS-axis clockwise rotation. Airway obstruction, RV afterload, RV/LV enddiastolic volume ratio and BMI correlated to the SokolowLyon Index for RV, and RV afterload negatively to SokolowLyon Index for LV. Several classical ECG changes could, however, not be ascribed to specific mechanisms.

Conclusions In COPD, the various pathophysiological mechanisms modify the ECG differently. Increased airway obstruction and RV afterload mainly increase the SokolowLyon Index for RV mass and associate with clockwise rotation of the horizontal QRS-axis, whereas emphysema reduces the QRS amplitudes. BMI is an equally important determinant for the majority of the ECG changes.

\section{INTRODUCTION}

Patients with chronic obstructive pulmonary disease (COPD) often have an abnormal ECG. There are various deviations from the normal, and no consistent pattern with worsening of airway obstruction. ${ }^{12}$ The number of deviations, however, increases with the Global Initiative for Chronic Obstructive Lung Disease (GOLD) class. ${ }^{3}$ The most consistent patterns reported have been vertical axes for $\mathrm{P}$ and $\mathrm{QRS},{ }^{145}$ often found in emphysema and increased P-wave amplitude (P-pulmonale). ${ }^{2467}$ The QRS amplitudes are often reduced ${ }^{12}$ (table 1$)$. However,

\section{KEY MESSAGES}

What is already known about this subject?

- The multitude of ECG changes in chronic obstructive pulmonary disease (COPD) has previously been well described, but the causes of the various ECG changes have not been in focus.

What does this study add?

- By extensive studies on a well characterised COPD population, associations between the ECG changes and the pathophysiological factors airway obstruction, emphysema and right ventricular afterload have been revealed by univariate and multivariate statistical analyses.

How might this impact on clinical practice?

- A better understanding of the ECG changes in COPD may improve interpretation of ECG in these patients and help revealing the dominant pathophysiology of their airway disease.

each abnormality is present in only a fraction of the patients, even in GOLD class 4, and the results vary between populations. ${ }^{2468}$

In some patients, airway obstruction dominates the clinical picture, in others emphysema. Pulmonary hypertension may result in hypertrophy and remodelling of the right side of the heart. Finally, body mass index (BMI), a possible confounder, decreases with worsening COPD. It is reasonable to assume that each of these pathophysiological mechanisms could differently modify the heart and the electrical conduction of the thorax. Previous studies have related ECG findings to obstruction (GOLD stage), ${ }^{12467}$ to emphysema (chest X-ray, ${ }^{8-10}$ autopsy) ${ }^{5}$ to increased pulmonary vascular pressure ${ }^{11-13}$ or right ventricular (RV) hypertrophy. ${ }^{14}$ However, none of these studies have linked their findings to the combined effects of obstruction, emphysema and increased afterload. Further, most previous studies have not excluded patients with the frequent concomitant left ventricular (LV) disease. Our aim was therefore to study ECG in patients with COPD 


\begin{tabular}{|c|c|}
\hline Emphysema & P-wave axis ${ }^{24}$ \\
\hline \multirow[t]{2}{*}{ Right-atrium enlargement ${ }^{2}$} & P-amplitude in II, III or aVF $\geq 2.5 \mathrm{~mm}^{12}$ \\
\hline & P-amplitude in V1 $\geq 1.5 \mathrm{~mm}^{2}$ \\
\hline \multirow[t]{3}{*}{ Scott et al ${ }^{11}$ : RVH } & $\mathrm{R}$ in $\mathrm{V} 1 \geq 7 \mathrm{~mm}$ \\
\hline & $\mathrm{R} / \mathrm{S}$ in $\mathrm{V} 1>1$ \\
\hline & VAT in $\mathrm{V} 1>35 \mathrm{~ms}$ \\
\hline Sokolow-Lyon: RVH & $\mathrm{R}$ in $\mathrm{V} 1+\mathrm{S}$ in $\mathrm{V} 5$ or $\mathrm{V} 6>10.5 \mathrm{~mm}^{16}$ \\
\hline Clockwise rotation & $\mathrm{R} / \mathrm{S}$ ratio in $\mathrm{V} 5 \leq 1^{1}$ \\
\hline Low voltage limb leads & QRS $(\mathrm{R}+\mathrm{S})<5 \mathrm{~mm}$ in I, II, aVF, III (all) ${ }^{\star 1}$ \\
\hline Low voltage precordial leads & $\mathrm{QRS}<10 \mathrm{mV}$ in $\mathrm{V} 1-\mathrm{V} 6$ (all) ${ }^{*}$ \\
\hline $\mathrm{S}_{1} \mathrm{~S}_{2} \mathrm{~S}_{3}$ pattern & Dominant S in I, II, III (all) ${ }^{1}$ \\
\hline QS complex & Lead III \\
\hline Right-axis deviation: & $>90^{\circ 1}$ \\
\hline Left-axis deviation: & $<-30^{\circ}$ to $-90^{\circ 12}$ \\
\hline Elevated resting heart rate & $\mathrm{HR}>80$ beats $/ \mathrm{min}^{*}$ \\
\hline
\end{tabular}

*Minnesota Code ${ }^{20}$

$\mathrm{HR}$, heart rate; $\mathrm{RVH}$, right ventricular hypertrophy; VAT, ventricular activation time.

and controls with a comprehensive armamentarium of non-invasive and invasive tests, and examine if it were possible to identify the influence from the underlying pathology on the ECG.

\section{METHODS \\ Materials}

This cross-sectional study recruited 112 outpatients with COPD of both sexes from Oslo University Hospital Aker during 2006-2010 ${ }^{15}$ : 40- to 75-yearold Norwegian Caucasians with at least 10 pack-years of cigarette smoking and irreversible airway obstruction demonstrated by spirometry. Participants were free from COPD exacerbations for the preceding 2 months, and graded to GOLD 2-4 by the classification of that time. ${ }^{3}$ Hospital employees with similar age distribution were used as controls (controls mean 63 years, range 40-76; COPD mean 64 years, range 41-77). In the analysis, the two groups are treated as independent.

Patients underwent standardised clinical examinations $^{12-14}$ during a 2-day visit including blood tests, spirometry, static lung volumes, exercise testing, ECG, echocardiography, CT and MRI. Neither the examinations nor their history suggested the presence of ischaemic heart disease. Significant other comorbidity was also excluded. ${ }^{12-14}$ Ninety-eight patients, but no controls, underwent rightheart catheterisation. Two control patients were excluded (missing ECGs). The study complies with the Declaration of Helsinki and was approved by the local research ethics committee. Written informed consent was obtained from all subjects.

\section{Methods}

\section{Electrocardiography}

Twelve-lead ECG (Schiller AG, Baar, Switzerland) was recorded after $10 \mathrm{~min}$ supine rest with paper speed of $50 \mathrm{~mm} / \mathrm{s}$, gain of $10 \mathrm{~mm} / \mathrm{mV}$ and default filter settings. ECGs were read manually by one investigator, and $20 \%$ randomly selected, and all ECGs with uncertain interpretation were read by two investigators; measures were decided by consensus. Right-atrium enlargement was defined when P-wave amplitude $\geq 2.5 \mathrm{~mm}$ in II, aVF or III or $\geq 1.5 \mathrm{~mm}$ in V1. RV hypertrophy was defined according to Scott $e t a l^{11}$ and Sokolow-Lyon, ${ }^{16}$ and LV hypertrophy according to Sokolow-Lyon ${ }^{17}$ and the Cornell voltage product (with the addition of $0.6 \mathrm{mV}$ for women), ${ }^{18}$ and used as an estimate for LV mass. The transition zone was the first precordial lead with either an isoelectric QRS or a configuration change from a dominant $\mathrm{S}(\mathrm{rS})$ to dominant R (Rs). Clockwise QRS-axis rotation (delayed transition) required $\mathrm{R} / \mathrm{S}$ transition at or lateral to $\mathrm{V} 4$, and counter-clockwise rotation was medial to V3. ${ }^{19}$ The Minnesota Code defined low voltage and axis deviations. ${ }^{20}$ A normal ECG required heart rate $50-80$ beats $/ \mathrm{min}, \mathrm{PQ}$ duration $0.12-0.20 \mathrm{~s}$, P-amplitude in $\mathrm{V} 1<1.5 \mathrm{~mm}$ and in II, aVF and $\mathrm{III}<2.5$, P-axis $<75^{\circ}$, QRS duration 0.07 $0.10 \mathrm{~s}$, at least one QRS amplitude in limb leads $\geq 5 \mathrm{~mm}$ and $\geq 10 \mathrm{~mm}$ in precordial leads, frontal QRS-axis $-30^{\circ}$ to $90^{\circ 21}$ and transition zone at V3.

\section{Echocardiography}

Echocardiography (Vivid 7; GE Vingmed Ultrasound, Horten, Norway) was performed with left parasternal long and short axes and apical four-chamber view adjusted to acquire the RV-focused view during breath hold at end expiration. Analyses were performed without knowledge of clinical status. RV end-diastolic and systolic areas were assessed by manual planimetry and divided by body surface area. RV wall thickness was obtained from the short-axis window by either M mode or two-dimensional echocardiography in end diastole. Real-time three-dimensional echocardiography was used to acquire full-volumetric datasets of the right and left ventricle from four ECG-triggered subvolumes. Postprocessing analysis (TomTec Imaging Systems GmbH, Germany) was performed with semi-automatic software with predefined RV views for endocardial contour delineation volumes. ${ }^{22}$

\section{Haemodynamic and pulmonary function}

Supine right-heart catheterisation (Mac-Lab; GE Healthcare, Milwaukee, Wisconsin, USA) was performed with 7F Swan-Ganz catheter as described in previous studies on these patients. ${ }^{152}$ Sitting upright, forced expiratory volume in $1 \mathrm{~s}\left(\mathrm{FEV}_{1}\right)$ and forced vital capacity (FVC) were determined by spirometry after bronchodilator, in accordance with international guidelines and Norwegian reference values,${ }^{153}$ using best values for GOLD classification. 
Table 2 Single and composite predictor variables and their correlations

\begin{tabular}{llllll}
\hline & GOLD & & \multicolumn{3}{c}{ Correlation } \\
\hline New variables & $2(n=40)$ & $3(n=30)$ & $4(n=31)$ & $t$ & $p^{*}$ \\
Obstruction (-FEV \%) & $-1.0(0.5)$ & $0.2(0.4)$ & $1.1(0.4)$ & 0.79 & $\mathbf{0 . 0 0 0 1}$ \\
Emphysema (residual volume \%, RV/TLC) & $-1.4(1.1)$ & $-0.1(1.9)$ & $1.9(1.1)$ & 0.56 & $\mathbf{0 . 0 0 0 1}$ \\
Afterload (mPAP, PVR,- PAC, RV wall) & $-1.5(1.7)$ & $-0.7(2.6)$ & $2.7(2.9)$ & 0.45 & $\mathbf{0 . 0 0 0 1}$ \\
\hline
\end{tabular}

${ }^{*} \mathrm{p}<0.05, \mathrm{p}$ values refer to Kendall's t correlation for trend between variables.

$\mathrm{FEV}_{1} \%$, forced expiratory volume in 1s \% predicted; GOLD, Global Initiative for Chronic Obstructive Lung Disease; mPAP, mean pulmonary artery pressure; PAC, pulmonary arterial compliance; PVR, pulmonary vascular resistance; RV/TLC, residual volume/total lung capacity; RV wall, right ventricle wall thickness; $t$, Kendalls t correlation.

\section{Statistical analysis}

To reduce the number of independent variables, haemodynamic and pulmonary function variables were grouped into three new variables: (1) airway obstruction expressed by $\mathrm{FEV}_{1} \%$ of predicted, (2) emphysema by residual volume/total lung capacity and residual volume $\%$ of predicted and (3) afterload by mean pulmonary artery pressure, pulmonary artery compliance, pulmonary vascular resistance and RV wall thickness.

These haemodynamic and pulmonary continuous variables were standardised to mean $=0$ and $\mathrm{SD}=1$. The converted variables in each group were added, and the sum represents the common score. For obstruction this was $-\mathrm{FEV}_{1} \%$ of the predicted, and thus increased value of an aggregated variable reflects increased disease burden (tables 2 and 3). Continuous variables are expressed as mean with SD, and categorical variables as frequencies or percentages. Kendall's tau rank correlation describes trend between variables and GOLD stage. Wilcoxon/ Kruskal-Wallis rank-sum test compares continuous variables for controls and COPD, and Fisher exact test evaluates categorical variables. A two-tailed $p$ value $<0.05$ was considered significant. Dependent variables that were not normally distributed by Shapiro-Wilk test were log-transformed and included in the regression models. To permit log-transformation, QRS-axis had to be expressed in positive values, and we thus redefined the variable to $\log 180$-(QRS-axis).

Multiple linear and logistic regression analyses were used to determine independent predictors of the ECG variables related to COPD (table 4). BMI, gender and the variables expressing airway obstruction, emphysema and afterload were entered in the full model. Age was not included in the model because there was no significant difference in age between the GOLD stages. Thereafter, backward stepwise eliminations were performed for non-significant $(p>0.05)$ variables. Statistical analyses were computed using JMP12.

\section{RESULTS}

Clinical characteristics are presented in table 5. Patients with COPD had significantly higher heart rate and blood pressure, compared with the controls. RV end-diastolic volume and wall thickness were significantly increased in patients. The ratio between LV and RV end-diastolic volume was significantly reduced in patients with COPD, following severity (GOLD stage). All indices for RV afterload increased with worsening of COPD.

\section{ECG measurements}

With increasing GOLD stage, P-wave amplitude in V1 lowered, Sokolow-Lyon Index describing LV mass was reduced, and QRS amplitudes were lower. There was also more clockwise and leftward change of QRS-axis (table 4). The prevalence of abnormal ECG was significantly higher in COPD compared with the control group.

\section{Univariate relationships between the ECG and independent variables}

Heart rate, but not P-axis, correlated positively to emphysema $(\mathrm{p}=0.02)$ (see online supplementary tables $\mathrm{S} 1$ and $\mathrm{S} 2)$. P-wave amplitude in $\mathrm{V} 1$ correlated positively to $\mathrm{RV} / \mathrm{LV}$

Table 3 Correlations between independent predictor variables

\begin{tabular}{|c|c|c|c|c|c|}
\hline & Gender & BMI & Obstruction & Emphysema & Afterload \\
\hline BMI & $0.2233^{*}$ & & & & \\
\hline Obstruction & -0.0480 & $-0.1733^{*}$ & & & \\
\hline Afterload & -0.1160 & $-0.1515^{\star}$ & $0.4132^{*}$ & $0.4214^{*}$ & \\
\hline RV/LV EDVI & -0.1339 & -0.0703 & $0.2417^{*}$ & $0.1546^{*}$ & $0.2417^{*}$ \\
\hline
\end{tabular}

Gender: female is a reference category for gender.

Bold values are statistically significant. Significant differences within each variable are indicated as:

${ }^{*} p<0.05 ; p$ values refers to Kendall's t correlation.

$\mathrm{BMI}$, body mass index; RV/LV EDVI, right ventricle/left ventricle end-diastolic volume ratio. 
Table 4 ECG measurements and prevalence of various ECG findings

\begin{tabular}{|c|c|c|c|c|c|c|}
\hline & Controls & GOLD & & & Controls vs COPD & GOLD stage \\
\hline ECG variables & $\mathrm{n}=32$ & $2(n=40)$ & $3(n=30)$ & $4(n=31)$ & $\mathbf{p}$ & $\mathbf{p}$ \\
\hline P-axis $\left(^{\circ}\right)$ & $52(21)$ & $67(26)$ & $64(41)$ & $69(22)$ & $<0.0001$ & 0.72 \\
\hline $\mathrm{P}$ in V1 $(\mathrm{mm})$ & $0.6(0.2)$ & $0.6(0.4)$ & $0.5(0.5)$ & $0.2(0.7)$ & 0.02 & 0.009 \\
\hline P in II, aVF, III (mm) & $1.3(0.5)$ & $1.3(0.6)$ & $1.3(0.8)$ & $1.3(0.8)$ & 0.92 & 0.94 \\
\hline QRS duration (ms) & $91(13)$ & 87 (16) & $88(15)$ & $83(12)$ & 0.09 & 0.46 \\
\hline QRS-axis $\left(^{\circ}\right)$ & $34(42)$ & $60(27)$ & $75(37)$ & $45(55)$ & 0.0002 & 0.94 \\
\hline V1 R/S & $0.2(0.2)$ & $0.4(0.6)$ & $0.5(0.9)$ & $0.2(0.2)$ & 0.98 & 0.38 \\
\hline V2 R/S & $0.6(0.4)$ & $0.8(1.3)$ & $0.6(0.9)$ & $0.4(0.5)$ & 0.02 & 0.02 \\
\hline V3 R/S & $2.0(3.1)$ & $1.8(2.3)$ & $1.3(1.2)$ & $1.0(1.6)$ & 0.02 & 0.02 \\
\hline V4 R/S & $5.7(6.4)$ & $4.4(7.5)$ & $4.2(6.2)$ & $2.8(3.8)$ & 0.007 & 0.10 \\
\hline V5 R/S & $10.2(11.4)$ & $6.5(7.4)$ & $6.2(7.4)$ & $4.1(5.0)$ & 0.02 & 0.02 \\
\hline SL RVH (mm) & $4(3)$ & $5(3)$ & $6(5)$ & $5(4)$ & 0.07 & 0.50 \\
\hline SL RVH p. (mm·ms) & $354(251)$ & 415 (286) & $524(479)$ & $451(325)$ & 0.17 & 0.66 \\
\hline SL LVH (mm) & $20(6)$ & $20(6)$ & $18(6)$ & $14(6)$ & 0.07 & 0.0003 \\
\hline Cornell Index (mm) & $13(6)$ & $12(5)$ & $12(5)$ & $15(6)$ & 0.98 & 0.12 \\
\hline Cornell p. (mm·ms) & $1243(734)$ & $1053(470)$ & $1070(504)$ & $1234(539)$ & 0.61 & 0.17 \\
\hline $\mathrm{R}+\mathrm{S}$ precordial leads & $81(20)$ & $80(18)$ & 77 (22) & 67 (19) & 0.13 & 0.01 \\
\hline $\mathrm{R}+\mathrm{S}$ frontal leads & $24(8)$ & $24(5)$ & $23(7)$ & $20(6)$ & 0.32 & 0.009 \\
\hline Precordial/frontal amp & $3.5(0.8)$ & $3.5(0.9)$ & $3.6(1.6)$ & $3.8(1.8)$ & 0.47 & 0.96 \\
\hline CCW n (\%) & $18(60)$ & $17(43)$ & $12(40)$ & $5(16)$ & 0.02 & 0.03 \\
\hline Clockwise n (\%) & $3(9)$ & $5(13)$ & $7(23)$ & $10(32)$ & 0.15 & 0.11 \\
\hline \multicolumn{7}{|c|}{ Prevalence of various ECG findings } \\
\hline Abnormal ECG & $8(25 \%)$ & $34(85 \%)$ & $20(67 \%)$ & $29(94 \%)$ & $<0.0001$ & 0.49 \\
\hline $\mathrm{HR}>80$ beats/min & $4(13 \%)$ & $24(60 \%)$ & $15(50 \%)$ & $22(71 \%)$ & $<0.0001$ & 0.44 \\
\hline P-axis: $<0^{\circ}$ or $>75^{\circ}$ & $1(3 \%)$ & $10(25 \%)$ & $10(34 \%)$ & $9(29 \%)$ & 0.0014 & 0.65 \\
\hline P-pulmonale & $0(0 \%)$ & $4(10 \%)$ & $2(7 \%)$ & $2(6 \%)$ & 0.18 & 0.57 \\
\hline QRS $<70$ or $>120 \mathrm{~ms}$ & $1(3 \%)$ & $12(30 \%)$ & $7(23 \%)$ & $5(16 \%)$ & 0.008 & 0.18 \\
\hline QRS-axis $-30^{\circ}$ to $90^{\circ}$ & $29(84 \%)$ & $40(100 \%)$ & $28(93 \%)$ & $24(77 \%)$ & 1.0000 & 0.001 \\
\hline Left axis $-31^{\circ}$ to $-90^{\circ}$ & $3(16 \%)$ & $0(0 \%)$ & $0(0 \%)$ & $5(16 \%)$ & 0.40 & 0.004 \\
\hline Right axis $>90^{\circ}$ & $0(0 \%)$ & $0(0 \%)$ & $2(7 \%)$ & $2(6 \%)$ & 1.0000 & 0.14 \\
\hline Low voltage I-III & $1(3 \%)$ & $0(0 \%)$ & $1(3 \%)$ & $1(3 \%)$ & 0.57 & 0.31 \\
\hline $\mathrm{R} / \mathrm{S}$ in $\mathrm{V} 1>1$ & $0(0 \%)$ & $1(3 \%)$ & $2(7 \%)$ & $3(10 \%)$ & 0.34 & 0.20 \\
\hline$R / S$ in $V 5<1$ & $0(0 \%)$ & $3(8 \%)$ & $3(10 \%)$ & $1(3 \%)$ & 0.19 & 0.57 \\
\hline $\mathrm{S}_{1} \mathrm{~S}_{2} \mathrm{~S}_{3}$ pattern & $2(7 \%)$ & $1(3 \%)$ & $2(8 \%)$ & $7(23 \%)$ & 0.73 & 0.009 \\
\hline RVH SL & $0(0 \%)$ & $0(0 \%)$ & $1(3 \%)$ & $3(10 \%)$ & 0.57 & 0.05 \\
\hline RVH Scott et al ${ }^{11}$ & $1(3 \%)$ & $2(5 \%)$ & $3(10 \%)$ & $2(6 \%)$ & 0.68 & 0.75 \\
\hline RVH Scott or SL ${ }^{1116}$ & $0(3 \%)$ & $3(8 \%)$ & $4(13 \%)$ & $1(4 \%)$ & 0.20 & 0.73 \\
\hline
\end{tabular}

Values are mean (SD) of continuous variables.

Bold values are statistically significant. p Values refer to Kendall's t- correlation for trend comparing GOLD stage II, III and IV for each variable and Wilcoxon/ Kruskal-Wallis Rank Sum Test comparing COPD and controls, with Chi square approximation.

amp, amplitudes; Cornell p, Cornell product; CCW, counter-clockwise; GOLD, Global Initiative for Chronic Obstructive Lung Disease; HR, heart rate; $p$, p value; SL RVH, Sokolow-Lyon right ventricular hypertrophy; SL RVH p, Sokolow-Lyon product; SL LVH, Sokolow-Lyon left ventricular hypertrophy.

end-diastolic volume ratio (EDV) $(\mathrm{p}=0.04)$. Both SokolowLyon for RVH $(\mathrm{p}=0.001)$ and leftward shift of the frontal QRS-axis $(p=0.01)$ correlated positively to BMI. Precordial amplitudes were negatively correlated to emphysema $(p=0.02)$, afterload $(p=0.05)$ and RV/LV EDV $(p=0.06)$. Frontal amplitudes were reduced with increased emphysema $(p=0.01)$ and afterload $(p=0.03)$. Sokolow-Lyon Index reflecting $\mathrm{LV}$ mass, correlated negatively to airway 
Table 5 Clinical characteristics of the study population

\begin{tabular}{|c|c|c|c|c|c|c|}
\hline & Controls & GOLD & & & $\begin{array}{l}\text { Controls vs } \\
\text { COPD }\end{array}$ & GOLD stage \\
\hline & $(n=32)$ & $2(n=40)$ & $3(n=30)$ & $4(n=31)$ & $\mathbf{p}$ & $\mathbf{p}$ \\
\hline Women/men & $18 / 14$ & $20 / 20$ & $15 / 15$ & $18 / 13$ & 0.84 & 0.53 \\
\hline Age (years) & $63(7)$ & $65(6)$ & $63(8)$ & $63(6)$ & 0.61 & 0.16 \\
\hline $\mathrm{BMI}\left(\mathrm{kg} / \mathrm{m}^{2}\right)$ & $25(3)$ & $25(4)$ & $25(5)$ & $22(6)$ & 0.76 & 0.02 \\
\hline Current smokers, n (\%) & $2(6)$ & $17(43)$ & $5(17)$ & $10(32)$ & $<0.0001$ & 0.26 \\
\hline HR (beats/min) & $66(12)$ & $81(17)$ & $81(16)$ & $88(18)$ & $<0.0001$ & 0.22 \\
\hline $\mathrm{SBP}(\mathrm{mm} \mathrm{Hg})$ & $121(17)$ & $144(21)$ & $136(21)$ & $137(23)$ & $<0.0001$ & 0.20 \\
\hline $\mathrm{DBP}(\mathrm{mm} \mathrm{Hg})$ & $76(13)$ & 70 (12) & $69(10)$ & $67(14)$ & 0.006 & 0.20 \\
\hline $\mathrm{mPAP}(\mathrm{mm} \mathrm{Hg})$ & - & $18(4)$ & $20(6)$ & $25(6)$ & - & $<0.0001$ \\
\hline $\mathrm{PAC}(\mathrm{mL} / \mathrm{mm} \mathrm{Hg})$ & - & $4.0(1.1)$ & $4.2(1.6)$ & $3.2(1.4)$ & - & 0.022 \\
\hline PVR (Wu) & - & $1.8(0.7)$ & $2.0(0.8)$ & $3.3(1.4)$ & - & $<0.0001$ \\
\hline $\mathrm{Cl}\left(\mathrm{L} / \mathrm{min} / \mathrm{m}^{2}\right)$ & - & $2.9(0.4)$ & $2.9(0.5)$ & $3.1(0.6)$ & - & 0.35 \\
\hline RV EDV (mL) & $107(22)$ & $126(34)$ & $137(39)$ & $121(27)$ & 0.001 & 0.67 \\
\hline LV EDV (mL) & $109(24)$ & $113(26)$ & $115(31)$ & $94(28)$ & 0.77 & 0.002 \\
\hline RV/LV EDVI & $1.0(0.1)$ & $1.1(0.2)$ & $1.2(0.2)$ & $1.3(0.3)$ & $<0.0001$ & $<0.0001$ \\
\hline RV wall thickness $(\mathrm{mm})$ & $3.5(0,5)$ & $5.2(0.8)$ & $5.8(1)$ & $6.5(1.2)$ & $<0.0001$ & $<0.0001$ \\
\hline $\mathrm{FEV}_{1}(\mathrm{~L})$ & $3.1(0.8)$ & $1.8(0.6)$ & $1.2(0.4)$ & $0.7(0.2)$ & $<0.0001$ & $<0.0001$ \\
\hline $\mathrm{FEV}_{1} / \mathrm{FVC}(\%)$ & $76(4)$ & $56(7)$ & $45(10)$ & $40(10)$ & $<0.0001$ & $<0.0001$ \\
\hline $\mathrm{FEV}_{1} \%$ predicted & - & $59(8)$ & $39(6)$ & $26(7)$ & - & $<0.0001$ \\
\hline DLCO \% predicted & - & $64(14)$ & $53(17)$ & $29(14)$ & - & $<0.0001$ \\
\hline Residual volume (L) & $2.6(0.4)$ & $4.6(0.7)$ & $4.5(1.2)$ & $5.6(1.2)$ & $<0.0001$ & $<0.0001$ \\
\hline Residual volume \% pred. & $118(16)$ & $161(32)$ & $205(64)$ & $262(47)$ & $<0.0001$ & $<0.0001$ \\
\hline $\operatorname{TLC}(\mathrm{L})$ & $6.9(1.3)$ & $6.9(1.2)$ & $7.3(1.4)$ & $7.8(1.6)$ & 0.39 & 0.02 \\
\hline TLC \% predicted & $112(11)$ & $117(15)$ & $125(28)$ & $137(20)$ & 0.002 & 0.0002 \\
\hline Residual volume/TLC (\%) & $39(5)$ & $52(8)$ & $61(11)$ & $72(5)$ & $<0.0001$ & $<0.0001$ \\
\hline $\mathrm{SaO}_{2}(\%)$ & - & $96(1)$ & $96(1)$ & $91(5)$ & - & $<0.0001$ \\
\hline $\mathrm{PaO}_{2}(\mathrm{kPa})$ & - & $10.1(1.0)$ & $9.7(1.2)$ & $7.9(1.3)$ & - & $<0.0001$ \\
\hline $\mathrm{PaCO}_{2}(\mathrm{kPa})$ & - & $5.1(0.4)$ & $5.4(0.6)$ & $6.0(0.8)$ & - & $<0.0001$ \\
\hline
\end{tabular}

Bold values are statistically significant.

BMI, body mass index; COPD, chronic obstructive pulmonary disease; DBP, diastolic blood pressure; DLCO, diffusion capacity for carbon monoxide of the lungs; $\mathrm{FEV}_{1} / \mathrm{FVC}$, forced expiratory volume in 1s/forced vital capacity; GOLD, Global Initiative for Chronic Obstructive Lung Disease; HR, heart rate; LV EDV, left ventricular end-diastolic volume; mPAP, mean pulmonary artery pressure; PAC, pulmonary arterial compliance; $\mathrm{PaO}_{2}$, arterial oxygen tension; $\mathrm{PaCO}$, arterial carbon dioxide tension; PVR, pulmonary vascular resistance; RV EDV, right ventricular end-diastolic volume; RV/LV EDVI, right ventricle/ left ventricle end-diastolic volume ratio; $\mathrm{SBP}$, systolic blood pressure; $\mathrm{SaO}$, arterial oxygen saturation; TLC, total lung capacity.

obstruction $(p=0.003)$, emphysema $(p=0.003)$, afterload $(\mathrm{p}=0.001)$ and the ratio $\mathrm{RV} / \mathrm{LV} \operatorname{EDV}(\mathrm{p}=0.06)$, whereas Cornell Index and product had a positive association to emphysema $(p=0.01)$ and afterload $(0.03)$, and were higher in men compared with women $(p=0.0001)$. Counter-clockwise rotation $(p=0.04)$ was negatively associated with afterload, and clockwise rotation positively with BMI $(\mathrm{p}=0.04)$.

\section{Multivariate regression analysis}

Multivariate linear regression analyses are shown in table 6 (and online supplementary table S3) and multivariate logistic regression in table 7 (and online supplementary table
S4), and were performed with gender, BMI, airway obstruction, emphysema, afterload and RV/LV EDV. For example, one SD increase of the independent variable emphysema corresponds to an increase in heart rate of 6 beats $/ \mathrm{min}$. Because of the high positive correlations between the variables afterload and obstruction in the regression models for Cornell Index and clockwise rotation, these variables had to be studied in separate regression models.

\section{DISCUSSION}

The present study is unique compared with previous COPD studies because of more extensive invasive and non-invasive 
Table 6 Independent predictors of ECG findings in patients with COPD

\begin{tabular}{|c|c|c|c|c|c|}
\hline ECG & Predictors & $\beta$-coefficient (SE) & $95 \% \mathrm{Cl}$ & p Value & $\mathbf{R}^{2}$ adj \\
\hline \multirow[t]{4}{*}{ Heart rate } & & & & & 0.04 \\
\hline & BMI & $0.3771(0.3890)$ & $(-0.3954$ to 1.1496$)$ & 0.34 & \\
\hline & Obstruction & $-2.4663(2.5794)$ & (-7.5879 to 2.6552$)$ & 0.34 & \\
\hline & Emphysema & 3.3934 (1.4087) & (0.5963 to 6.1905$)$ & 0.02 & \\
\hline \multirow[t]{6}{*}{ Log (P-wave V1) } & & & & & 0.08 \\
\hline & BMI & $-0.0083(0.0120)$ & $(-0.0323$ to 0.0156$)$ & 0.49 & \\
\hline & Obstruction & $-0.1431(0.0721)$ & $(-0.2870$ to 0.0007$)$ & 0.05 & \\
\hline & Emphysema & $0.0638(0.0420)$ & (-0.01994 to 0.1476$)$ & 0.13 & \\
\hline & Afterload & $-0.0311(0.0214)$ & $(-0.0738$ to 0.0115$)$ & 0.15 & \\
\hline & RV/LV EDVI & $0.6196(0.2190)$ & (0.1832 to 1.0561$)$ & 0.006 & \\
\hline \multirow[t]{4}{*}{ Log (QRS duration) } & & & & & 0.06 \\
\hline & Gender* & $-0.0311(0.0166)$ & (-0.0641 to 0.0017$)$ & 0.06 & \\
\hline & Afterload & $0.0084(0.0060)$ & $(-0.0036$ to 0.0204$)$ & 0.17 & \\
\hline & RV/LV EDVI & $-0.1458(0.0744)$ & (-0.2936 to 0.0020$)$ & 0.05 & \\
\hline \multicolumn{6}{|l|}{$\log (180-Q R S \text { axis })^{\dagger}$} \\
\hline & Gender* & $0.0309(0.0282)$ & $(-0.0251$ to 0.0868$)$ & 0.28 & \\
\hline & BMI & $0.0170(0.0059)$ & (0.0020 to 0.0288 ) & 0.005 & \\
\hline & Obstruction & $0.0382(0.0288)$ & $(-0.0190$ to 0.0954$)$ & 0.19 & \\
\hline & RV/LV EDVI & $-0.1579(0.1187)$ & $(-0.3935$ to 0.0777$)$ & 0.19 & \\
\hline \multirow[t]{5}{*}{$\mathrm{R}+\mathrm{S}$ precordial leads } & & & & & 0.07 \\
\hline & Obstruction & $5.8220(3.1490)$ & $(-0.4371$ to 12.0811$)$ & 0.08 & \\
\hline & Emphysema & $-3.7882(1.6900)$ & $(-7.1473$ to -0.4291$)$ & 0.03 & \\
\hline & Afterload & $-0.1184(0.9034)$ & $(-1.9140$ to 1.6772$)$ & 0.90 & \\
\hline & RV/LV EDVI & -15.9687 (9.1503) & (-34.1560 to 2.2187 ) & 0.09 & \\
\hline \multirow[t]{7}{*}{$\mathrm{R}+\mathrm{S}$ frontal leads } & & & & & 0.11 \\
\hline & Gender* & $0.8272(0.6782)$ & (-0.5214 to 2.1757$)$ & 0.23 & \\
\hline & BMI & $-0.3702(0.1447)$ & $(-0.6580$ to -0.0823$)$ & 0.01 & \\
\hline & Obstruction & $1.4496(1.0280)$ & $(-0.5944$ to 3.4936$)$ & 0.16 & \\
\hline & Emphysema & $-1.5747(0.6008)$ & $(-2.7693$ to -0.3800$)$ & 0.01 & \\
\hline & Afterload & $-0.1662(0.2817)$ & $(-0.7263$ to 0.3939$)$ & 0.56 & \\
\hline & RV/LV EDVI & $-3.2500(2.8746)$ & $(-8.9655$ to 2.4656$)$ & 0.26 & \\
\hline \multirow[t]{7}{*}{ Log (Sokolow-Lyon RVH) } & & & & & 0.19 \\
\hline & Gender* & $-0.0453(0.0708)$ & $(-0.1861$ to 0.0956$)$ & 0.52 & \\
\hline & BMI & $0.0383(0.0151)$ & (0.0082 to 0.0684$)$ & 0.01 & \\
\hline & Obstruction & $0.2523(0.1074)$ & (0.0388 to 0.4657$)$ & 0.02 & \\
\hline & Emphysema & $-0.1090(0.0627)$ & $(-0.2338$ to 0.0157$)$ & 0.09 & \\
\hline & Afterload & $0.0825(0.0294)$ & (0.0241 to 0.1411$)$ & 0.006 & \\
\hline & RV/LV EDVI & $-0.6141(0.3002)$ & $(-1.2110$ to -0.0172$)$ & 0.04 & \\
\hline \multicolumn{2}{|c|}{ Log (Sokolow-Lyon RVH product) } & & & & 0.20 \\
\hline & Gender* & $-0.0720(0.0761)$ & $(-0.2235$ to 0.0795$)$ & 0.35 & \\
\hline & BMI & $0.0389(0.0162)$ & (0.0065 to 0.0712$)$ & 0.02 & \\
\hline & Obstruction & $0.2560(0.1155)$ & (0.0264 to 0.4856$)$ & 0.03 & \\
\hline & Emphysema & $-0.1180(0.0675)$ & $(-0.2521$ to 0.0162$)$ & 0.08 & \\
\hline & Afterload & $0.0947(0.0317)$ & (0.0317to 0.1575$)$ & 0.004 & \\
\hline
\end{tabular}


Pulmonary vascular disease

Table 6 Continued

\begin{tabular}{|c|c|c|c|c|c|}
\hline \multirow[t]{2}{*}{ ECG } & Predictors & $\beta$-coefficient (SE) & $95 \% \mathrm{Cl}$ & p Value & $\mathbf{R}^{2}$ adj \\
\hline & RV/LV EDVI & $-0.7719(0.3229)$ & $(-1.4140$ to -0.1298$)$ & 0.02 & \\
\hline \multicolumn{5}{|c|}{ Sokolow Lyon left ventricular hypertrophy } & 0.08 \\
\hline & Gender* & $-0.5269(0.6870)$ & $(-1.8927$ to 0.8388$)$ & 0.45 & \\
\hline & BMI & $-0.1964(0.1482)$ & (-0.4912 to 0.0983$)$ & 0.19 & \\
\hline & Obstruction & $-0.3134(1.0412)$ & $(-2.3834$ to 1.7565$)$ & 0.76 & \\
\hline & Emphysema & $-0.3042(0.6073)$ & $(-1.5156$ to 0.9030$)$ & 0.62 & \\
\hline & Afterload & $-0.5327(0.2660)$ & $(-1.0615$ to -0.0038$)$ & 0.05 & \\
\hline \multicolumn{5}{|c|}{ Cornell Index model 1} & 0.34 \\
\hline & Gender* & $3.2567(0.4886)$ & (2.2860 to 4.2275$)$ & $<0.0001$ & \\
\hline & $\mathrm{BMI}$ & $0.1759(0.1030)$ & $(-0.0288$ to 0.3807$)$ & 0.09 & \\
\hline & Afterload & $0.3542(0.1778)$ & (0.0009 to 0.7076$)$ & 0.05 & \\
\hline & RV/LV EDVI & $-0.2416(2.1360)$ & $(-4.4851$ to 4.0019$)$ & 0.91 & \\
\hline \multicolumn{5}{|c|}{ Cornell Index model 2} & 0.34 \\
\hline & Gender* & $3.3363(0.4697)$ & (2.4037 to 4.2687 ) & $<0.0001$ & \\
\hline & BMI & $0.1697(0.0992)$ & $(-0.0272$ to 0.0366$)$ & 0.09 & \\
\hline & Obstruction & $0.9709(0.4815)$ & (0.0150 to 1.9267$)$ & 0.05 & \\
\hline & RV/LV EDVI & $0.2188(1.9797)$ & $(-3.7109$ to 4.1486$)$ & 0.91 & \\
\hline
\end{tabular}

Multiple linear regression analysis.

Bold values are statistically significant. $p$ Value refers to t-test of regression coefficient.

${ }^{*}$ Female as reference category. †Log (180-QRS axis): increased value reflects a more leftward axis.

$\mathrm{R}^{2}$ adj, adjusted $\mathrm{R}^{2}$; BMI, body mass index; COPD, chronic obstructive pulmonary disease; RV/LV EDVI, right ventricle/left ventricle end-

diastolic volume ratio; $\mathrm{RVH}$, right ventricular hypertrophy.

examinations, allowing us to demonstrate that three pathophysiological COPD components have major impacts on ECG. However, even among the most severely affected patients in GOLD class IV, a minority had a normal ECG. Since patients with COPD often have LV disease with accompanying ECG changes, we emphasise that our COPD

Table 7 Independent predictors for horizontal clockwise rotation of the QRS-axis

\begin{tabular}{|c|c|c|c|c|c|c|}
\hline ECG & $\begin{array}{l}\text { Independent } \\
\text { variable }\end{array}$ & $\beta$ & OR & $95 \% \mathrm{Cl}$ & p Value & $R^{2}$ adj \\
\hline \multirow[t]{3}{*}{ Model 1} & & & & & & 0.18 \\
\hline & $\mathrm{BMI}$ & 0.1465 & 1.1578 & (1.0277 to 1.3238 ) & 0.02 & \\
\hline & Obstruction & 0.6057 & 1.8325 & (0.7400 to 4.8112$)$ & 0.20 & \\
\hline \multirow[t]{4}{*}{ Model 2} & & & & & & 0.16 \\
\hline & Gender* & -0.0739 & 1.1594 & (0.4064 to 3.2821 ) & 0.78 & \\
\hline & BMI & 0.1331 & 1.1424 & (1.0251 to 1.2867 ) & 0.02 & \\
\hline & Obstruction & 0.5674 & 1.7638 & (1.0425 to 3.1681 ) & 0.04 & \\
\hline Model 3 & Afterload & 0.1894 & 1.2085 & (1.0103 to 1.4622$)$ & 0.04 & \\
\hline
\end{tabular}

Multiple logistic regression analysis.

Bold values are statistically significant. $p$ Values refer to Likelihood ratio Chi square test of the regression estimates.

${ }^{\star}$ Female as reference category.

$\beta$, regression coefficient; $\mathrm{R}^{2}$ adj, adjusted $\mathrm{R}$-square; BMI, body mass index; RV/LV EDVI, right ventricle end-diastolic volume ratio; RVH, right ventricular hypertrophy. 
population was recruited from clinically stable outpatients, where LV disease as well as other comorbidities that might affect the ECG, had been thoroughly excluded. ${ }^{15223}$

The patients showed typical clinical characteristics (table 2), and their ECG changes were mostly consistent with previous studies. ${ }^{24}{ }^{25}$ Exceptions were the low prevalence of right atrium enlargement and RV hypertrophy in ECG, in contrast to Holzmann and coworkers. ${ }^{2}$ Sixtythree percent of our patients had abnormal ECG, versus $25 \%$ of the controls. The controls had, however, only minor, clinically insignificant deviations from normal ECGs (table 3). The study confirms that COPD patients have higher heart rate than controls, more inferior $\mathrm{P}$-axis and more inferior, clockwise rotated QRS.

A vast number of anatomical and pathophysiological variables correlated with one or more ECG parameters in univariate analyses. Because their effects often were overlapping (ie, pulmonary artery pressure and pulmonary vascular resistance), significant correlations often disappeared in multivariate analyses. In order to reduce the number of variables that might affect the ECGs, without losing much information, we organised them into three main groups that were expected to influence the ECG: airway obstruction, emphysema and RV afterload. The variables were standardised, allowing us to add them into one of the three group variables. These correlated highly significantly (table 4), but not to a degree that precludes analysis of the separate effects of each one (tables 6 and 7). However, sometimes it was difficult to separate the effects on some of the ECG signs. For example, when studying clockwise rotation or heart rate, we were unable to separate the effects of obstruction and afterload on the dependent variable. Table 6 shows that although correlations were highly significant, the $\mathrm{R}^{2}$ values were rather low, attesting to the complexity of the relationships between the pathophysiological mechanisms.

\section{ECG changes related to the three underlying pathophysiological mechanisms \\ Obstruction}

Only $8 \%$ of our COPD patients had the classical P-pulmonale, in contrast to previous studies, ${ }^{46}$ and we saw P-wave lowering in V1 with increasing GOLD stage (table 3). Our patients with P-pulmonale did not have elevated right atrial pressure, confirming previous studies that related this sign to a vertical heart. ${ }^{13}{ }^{14} \mathrm{P}$-axis has been suggested as a marker of emphysema in COPD,${ }^{24}$ but we could not separate the effects of airway obstruction and emphysema. In an old autopsy study, no patient with pure emphysema without chronic airway obstruction had abnormal P-axis. ${ }^{5}$ Hyperinflation due to obstruction or emphysema isolates electrically, ${ }^{2}$ lowers and verticalises the heart, resulting in clockwise rotation; all effects that reduce P-amplitude in V1. This amplitude increased when the ratio RV/LV EDV increased (rotation by RV expansion, increased electrical forces), even when adjusted for BMI, emphysema and afterload. In GOLD 4, LV EDV was reduced (table 2), probably due to increased pulmonary vascular resistance (hypoxia-induced vasoconstriction), favouring further clockwise rotation. As expected, increased airway obstruction correlated positively to Sokolow-Lyon Index for RV mass (adjusted for gender, BMI, emphysema and afterload). There was, however, a high correlation between the variables afterload and obstruction. The reduced Sokolow-Lyon Index for LV mass by obstruction $(\mathrm{p}=0.0034)$ and afterload $(\mathrm{p}=0.0014)$ (univariate analyses) presumably reflects both increased rightsided and decreased left-sided QRS amplitudes by the combined anatomical and electrical remodelling of the heart.

\section{Emphysema}

We chose the composite variable residual volume/total lung capacity and residual volume percent predicted to represent emphysema. Diffusion capacity for carbon monoxide was not useful because of missing data, mainly from patients in GOLD 4. Univariate analyses showed negative correlations between emphysema and LV mass (QRS amplitudes, Sokolow-Lyon and Cornell Index and product for LV hypertrophy). On multivariable adjustment, only heart rate and reduced QRS amplitudes were significantly related to increased emphysema (table 7). Reduced QRS voltage in emphysema is established knowledge, ${ }^{18}$ and several mechanisms are involved. Increased electrical resistance due to air-filled bullae is a main contributor. The heart descends (hyperinflation and flattening of the diaphragm), reducing the anterior electrical forces at the level of the precordial electrodes. Clockwise and apical backward rotation contribute further, ${ }^{9}$ as does remodelling because LV filling is impaired. ${ }^{26} 27$

We could not confirm previous reports of significantly shorter QRS duration in COPD, ${ }^{24}{ }^{28}$ but found a borderline significant negative correlation between the ratio RV/LV EDV and QRS duration, adjusted for gender and afterload (table 6). The reasons for QRS narrowing are unknown.

Low voltage in limb leads, a posterior (horizontal QRS-axis $-50^{\circ}$ or beyond) and deviant frontal QRS-axis $\left(>70^{\circ}\right.$ or superior beyond $\left.-30^{\circ}\right)$, combined with a P-axis $>60^{\circ}$, are reported to be pathognomonic for emphysema. ${ }^{8}$ In the present study, $21 \%$ of the patients fulfilled these criteria, which were significantly correlated to obstruction, but not to emphysema.

\section{Afterload}

To assess RV afterload, we used the composite of the haemodynamic variables pulmonary vascular resistance, mean pulmonary artery pressure, pulmonary arterial compliance and the morphological consequence, RV wall thickness. In the literature, RV hypertrophy associates with right-axis deviation, dominant $\mathrm{R}$ in $\mathrm{V} 1$ and dominant $\mathrm{S}$ in V5-6. Only $12 \%$ of our patients satisfied the ECG criteria for RV hypertrophy. In contrast, Holzmann and coworkers reported a high prevalence of ECG signs for right-atrium enlargement and RV hypertrophy. ${ }^{2}$ In our 
study, ECG signs for RV hypertrophy t were significantly more prevalent in patients with pulmonary hypertension (mPAP $>25 \mathrm{~mm} \mathrm{Hg}$ ), in accordance with Johnson et al. ${ }^{11} 12$ As expected, increased afterload correlated to increased Sokolow-Lyon Index for RV hypertrophy, and reduced left-sided Sokolow-Lyon Index after adjustment for BMI, gender, obstruction, emphysema and RV/LV EDV.

Afterload increased the odds for clockwise rotation in the horizontal plane when we adjusted for BMI and gender. The patients with clockwise rotation had increased pressure in the pulmonary artery, increased pulmonary vascular resistance, a higher proportion of patients in GOLD 4 and increased air trapping and hyperinflation. They were also poorly oxygenated. Rotation of the heart in the horizontal plane was studied by Tahara and coworkers. They related the transition zone (precordial $\mathrm{R}>\mathrm{S}$ ) to the anatomical position of the septum as shown by CT scan, ${ }^{19}$ and demonstrated that anatomy was responsible for $2 / 3$ of the location forces. An enlarged right ventricle and vertical displacement of the heart due to obstruction and emphysema, favour clockwise rotation. Hypertrophy of the RV increases the initial rightward forces in ( $\mathrm{R}$ in $\mathrm{V} 1-\mathrm{V} 2)$, moving the transition counter clockwise. However, an increased $\mathrm{S}$ in v4-v6 (terminal rightward force) may be large enough to move the transition clockwise, as frequently observed in our patients.

RV afterload was negatively associated with SokolowLyon Index for LV mass, but positively associated with Cornell Index and product when adjusted for gender and BMI. This seems to be a paradox but may be ascribed to axis deviation reducing anterior QRS forces (SokolowLyon) as clockwise rotation of the heart moves the axis upward, posterior and to the left, as detected by the Cornell criteria.

\section{Gender and BMI}

Patients with advanced COPD are often cachectic. Fortyfive percent of our patients, however, had BMI $>25 \mathrm{~kg} /$ $\mathrm{m}^{2}$, and there was a wide range. We therefore included not only gender but also BMI in the analysis of the study population, since both variables influence the ECG measurements ${ }^{21} 29$ and the pathophysiological changes in COPD. ${ }^{30}$ We could confirm the normal gender ${ }^{21}$ and BMI $^{29}$ influence on ECG, and also that BMI correlated positively to Sokolow-Lyon Index for RV hypertrophy, and to a more leftward, clockwise rotated QRS-axis (tables 6 and 7$)$.

\section{Limitations}

The study population had moderate size, but we consider the study large due to the broad panel of examinations. Patients with signs of established left-sided cardiovascular disease, common in COPD, were thoroughly excluded. Thus, our study population is unique for analysis of pathophysiological factors that influence ECG, but not necessarily for patients with COPD in general. Unfortunately, data from high-resolution CT were not available for quantifying emphysema, so we used residual volume\% and RV/TLC to grade emphysema.

\section{CONCLUSIONS}

Aggregation of data from echocardiography, heart catheterisation and spirometryallowed us to relate ECG patterns in COPD to the separated, graded effects of emphysema, airway obstruction and RV afterload. Increased airway obstruction and RV afterload were mainly associated with increased Sokolow-Lyon Index for RV mass and clockwise rotation of the horizontal QRS-axis, and emphysema with increased heart rate and reduced QRS amplitudes. P-axis was not a good marker for emphysema in this study, but a low $\mathrm{P}$ in v1 might suggest airway obstruction. BMI seemed to be an equally important determinant for the majority of the ECG changes. These analyses may explain the heterogeneity of the ECG patterns in COPD. Some classical ECG features of COPD could not be ascribed to one pathophysiological mechanism, so still, when suspecting ECG changes in COPD, we shall look for a modest increase in heart rate, a vertical $\mathrm{P}$-axis, a small $\mathrm{P}$-wave in v1, small QRS amplitudes, a QRS-axis that is vertical or slightly deviant (usually to the left) and clockwise rotation of the precordial (horizontal) QRS transition zone.

Acknowledgements Morten Nissen Melsom, MD, and Sjur Humerfelt, MD, are gratefully acknowledged for supervising the pulmonary function tests and Viggo Hansteen, MD, for supervising the heart catheterisations.

Contributors MSL collected data, wrote the statistical analysis plan, analysed the data and drafted and revised the paper. KS collected data, drafted and revised the paper. JMH collected data, drafted and revised the paper. IS collected data, drafted and revised the paper. $\mathrm{CH}$ collected data, drafted and revised the paper. KL wrote the statistical analysis plan, analysed the data and revised the draft paper. KG wrote the statistical analysis plan, analysed the data and drafted and revised the paper.

Competing interests None declared.

Provenance and peer review Not commissioned; internally peer reviewed. Data sharing statement The authors accept data sharing on request.

Open Access This is an Open Access article distributed in accordance with the Creative Commons Attribution Non Commercial (CC BY-NC 4.0) license, which permits others to distribute, remix, adapt, build upon this work non-commercially, and license their derivative works on different terms, provided the original work is properly cited and the use is non-commercial. See: http://creativecommons.org/ licenses/by-nc/4.0/

(C) Article author(s) (or their employer(s) unless otherwise stated in the text of the article) 2017. All rights reserved. No commercial use is permitted unless otherwise expressly granted.

\section{REFERENCES}

1. Rodman DM, Lowenstein SR, Rodman T. The electrocardiogram in chronic obstructive pulmonary disease. J Emerg Med 1990;8:607-15.

2. Holtzman D, Aronow WS, Mellana WM, et al. Electrocardiographic abnormalities in patients with severe versus mild or moderate chronic obstructive pulmonary disease followed in an academic outpatient pulmonary clinic. Ann Noninvasive Electrocardiol 2011;16:30-2.

3. Rabe KF, Hurd S, Anzueto A, et al; Global Initiative for Chronic Obstructive Lung Disease. Global strategy for the diagnosis, management, and prevention of chronic obstructive pulmonary disease: GOLD executive summary. Am J Respir Crit Care Med 2007;176:532-55.

4. Calatayud JB, Abad JM, Khoi NB, et al. P-wave changes in chronic obstructive pulmonary disease. Am Heart J 1970;79:444-53. 
5. Shmock CL, Pomerantz B, Mitchell RS, et al. The electrocardiogram in emphysema with and without chronic airways obstruction. Chest 1971;60:328-34.

6. Spodick DH, Hauger-Klevene JH, Tyler JM, et al. The electrocardiogram in pulmonary emphysema. Am Rev Respir Dis 1963;88:14-19.

7. Kamper D, Chou TC, Fowler NO, et al. The reliability of electrocardiographic criteria of chronic obstructive lung disease. Am Heart J 1970;80:445-52.

8. Selvester RH, Rubin HB. New criteria for the electrocardiographic diagnosis of emphysema and cor pulmonale. Am Heart $J$ 1965;69:437-47.

9. Zuckermann R, Enrique CC, Fishleder BL, et al. Electrocardiogram in chronic cor pulmonale. Am Heart J 1948;35:421-37.

10. Chhabra L, Sareen P, Gandagule A, et al. Visual computed tomographic scoring of emphysema and its correlation with its diagnostic electrocardiographic sign: the frontal $\mathrm{P}$ vector. $J$ Electrocardiol 2012;45:136-40.

11. Scott RC, Kaplan S, Fowler NO, et al. The electrocardiographic pattern of right ventricular hypertrophy in chronic cor pulmonale. Circulation 1955;11:927-36.

12. Johnson JB, Ferrer MI, West JR, et al. The relation between electrocardiographic evidence of right ventricular hypertrophy and pulmonary arterial pressure in patients with chronic pulmonary disease. Circulation 1950;1:536-50.

13. Maeda S, Katsura H, Chida K, et al. Lack of correlation between $\mathrm{P}$ pulmonale and right atrial overload in chronic obstructive airways disease. Br Heart J 1991;65:132-6.

14. Murphy ML, Hutcheson F. The electrocardiographic diagnosis of right ventricular hypertrophy in chronic obstructive pulmonary disease. Chest 1974;65:622-7.

15. Hilde JM, Skjørten I, Hansteen V, et al. Haemodynamic responses to exercise in patients with COPD. Eur Respir J 2013;41:1031-41.

16. Sokolow M, Lyon TP. The ventricular complex in right ventricular hypertrophy as obtained by unipolar precordial and limb leads. Am Heart J 1949;38:273-94.

17. Sokolow M, Lyon TP. The ventricular complex in left ventricular hypertrophy as obtained by unipolar precordial and limb leads. Am Heart J 1949;37:161-86.
18. Dahlöf B, Devereux RB, Kjeldsen SE, et al; LIFE Study Group. Cardiovascular morbidity and mortality in the Losartan Intervention for Endpoint reduction in hypertension study (LIFE): a randomised trial against atenolol. Lancet 2002;359:995-1003.

19. Tahara $\mathrm{Y}$, Mizuno H, Ono A, et al. Evaluation of the electrocardiographic transitional zone by cardiac computed tomography. J Electrocardiol 1991;24:239-45.

20. Wright-PSG J. The Minnesota Code manual of electrocardiographic findings. MA: Inc Littleton, 1982.

21. Rijnbeek PR, van Herpen G, Bots ML, et al. Normal values of the electrocardiogram for ages $16-90$ years. $J$ Electrocardiol 2014:47:914-21.

22. Hilde JM, Skjørten I, Grøtta OJ, et al. Right ventricular dysfunction and remodeling in chronic obstructive pulmonary disease without pulmonary hypertension. J Am Coll Cardiol 2013;62:1103-11.

23. Skjørten I, Hilde JM, Melsom MN, et al. Pulmonary artery pressure and $\mathrm{PaO} 2$ in chronic obstructive pulmonary disease. Respir Med 2013;107:1271-9.

24. Thomas AJ, Apiyasawat S, Spodick DH. Electrocardiographic detection of emphysema. Am J Cardiol 2011;107:1090-2.

25. Warnier MJ, Rutten FH, Numans ME, et al. Electrocardiographic characteristics of patients with chronic obstructive pulmonary disease. COPD 2013;10:62-71.

26. Jörgensen K, Müller MF, Nel J, et al. Reduced intrathoracic blood volume and left and right ventricular dimensions in patients with severe emphysema: an MRI study. Chest 2007:131:1050-7.

27. Smith BM, Prince MR, Hoffman EA, et al. Impaired left ventricular filling in COPD and emphysema: is it the heart or the lungs? the Multi-Ethnic Study of Atherosclerosis COPD Study. Chest 2013;144:1143-51.

28. Zambrano SS, Moussavi MS, Spodick DH. QRS duration in chronic obstructive lung disease. J Electrocardiol 1974;7:35-6.

29. Fraley MA, Birchem JA, Senkottaiyan N, et al. Obesity and the electrocardiogram. Obes Rev 2005;6:275-81.

30. Camp PG, O'Donnell DE, Postma DS. Chronic obstructive pulmonary disease in men and women: myths and reality. Proc Am Thorac Soc 2009;6:535-8. 